\title{
Modulational instability of bright solitary waves in incoherently coupled nonlinear Schrödinger equations
}

\author{
Dmitry V. Skryabin *, and William J. Firth \\ Department of Physics and Applied Physics, John Anderson Building, \\ University of Strathclyde, 107 Rottenrow, Glasgow, G4 0NG, Scotland
}

(October 5, 1998)

We present a detailed analysis of the modulational instability (MI) of ground-state bright solitary solutions of two incoherently coupled nonlinear Schrödinger equations. Varying the relative strength of cross-phase and self-phase effects we show existence and origin of four branches of MI of the twowave solitary solutions. We give a physical interpretation of our results in terms of the group velocity dispersion (GVD) induced polarization dynamics of spatial solitary waves. In particular, we show that in media with normal GVD spatial symmetry breaking changes to polarization symmetry breaking when the relative strength of the cross-phase modulation exceeds a certain threshold value. The analytical and numerical stability analyses are fully supported by an extensive series of numerical simulations of the full model.

\section{INTRODUCTION}

The phenomenon of modulational instability (MI) can be defined as self-induced break up of an initially homogeneous wave during its evolution in a nonlinear medium. Study of this phenomenon has been initiated in the 60's, when MI was predicted in plasma physics [1], nonlinear optics [2] and physics of fluids [3], and also observed experimentally in form of filamentation of an optical beam propagating in an organic liquid [4]. Since that time MI has remained as one of the major topics of theoretical and experimental research in nonlinear physics and, in particular, in nonlinear physics of conservative systems [5 9,11. We will deal below with one classical example of such systems, unifying a number of previous results, and presenting new MI phenomena. Our approach stresses the central role of symmetries.

A general formulation of the problem of nonlinear wave propagation via fundamental sets of equations, as for example, the Maxwell or Navier Stokes equations, is a very demanding task even for modern computers. Therefore a number of simplified models have been introduced which approximately describe either propagation of the wave itself, e.g., KdV equation [12], or propagation of slowly varying wave envelope, e.g., the Nonlinear Schrödinger (NLS) equation [12].

The simplest solutions of envelope equations are continuous wave $(\mathrm{CW})$ solutions homogeneous in space and time. CWs in single NLS equation exhibit MI in cases when nonlinearity and group velocity dispersion (GVD) or diffraction act in opposition, e.g. when nonlinearity is positive GVD must be anomalous and if nonlinearity is negative GVD must be normal. This rule changes when, accounting for polarization, for different directions of wave vectors, or for different carrier frequencies of the interacting waves, one replaces the single NLS by the set of incoherently coupled NLS equations. Then, if nonlinear coupling is strong enough, MI of CWs becomes possible for any signs of nonlinearity and GVD 13 20].

Another important class of solutions of nonlinear equations are solitary solutions ('solitons'). They may also exhibit MI if they are localized in some dimensions but extended in one or more others. MIs of the envelope solitons of the single NLS and of the KdV solitons were pioneered, respectively, by Zakharov and Rubenchik 21] and by Kadomtsev and Petviashvily 22]. Latter MI was studied in a number of other theoretical and experimental works. For reviews on MI of bright and dark solitary waves see, respectively, 610 and 10,11.

From a formal point of view the problem of the solitary wave MI can be considered as continuation of the soliton spectrum at zero modulational frequency $\Omega$ into the region $\Omega \neq 0$. An important class of discrete eigenmodes at $\Omega=0$ are the zero eigenvalue (or neutral) modes. Presence of which is directly linked to symmetries of the model equations. On a qualitative level, similarities and differences between MI of solitons and CW solutions can be understood on the basis of a comparison between the corresponding neutral modes. For example, the 1D, bright spatial soliton of NLS is modulationally unstable in media with either anomalous or normal GVD. In first case, the neutral mode associated with the phase symmetry is excited ('neck' MI) and in latter situation the translational mode associated with a shift along the direction perpendicular to the wave propagation becomes unstable ('snake' MI) 21]. The phase mode is present as well for CW solution and this leads to MI for anomalous GVD. However, the translational mode of CW solution is null and therefore CWs are stable for normal GVD.

Increasing the number of free parameters can lead to more complex scenarios of MI, because coexistence and competition between different types of instability are likely to happen. In a recent Letter 23 we considered GVD induced MI of spatial solitons due to nondegenerate three-wave mixing. It was shown that presence of an additional phase symmetry leads to the appearance of a new branch of neck MI in media with normal GVD. It was found that the novel instability strongly dominates 
the usual snake MI throughout the region of soliton existence. Note, that because of this dominance, physical mechanisms responsible for the relative strength of neck and snake instabilities remain to be understood. Among others, this last problem is addressed in the present work, where we study MI of the bright solitary solutions in the incoherently coupled NLS equations. The incoherent nature of the coupling results in the presence of two phase symmetries. In spite of the similar symmetry properties, the features of MI induced dynamics of solitons in the present model appear to be more rich compare to the three-wave mixing case. In particular we show that the relative strength of the nonlinear cross-coupling governs the competition between neck and snake MIs in media with normal GVD.

The rest of this paper is organized as follows. In Section 2 we introduce model equations and discuss their physical relevance. In Section 3 the problem of MI of the solitary waves is formulated in general terms. MI of different kinds of solitary solutions and its physical interpretation in terms of polarization dynamics are detailed in Sections 4 and 5. Discussion and summary of main results are presented in Sections 6 and 7 .

\section{APPLICATIONS OF INCOHERENTLY COUPLED NLS EQUATIONS TO PROPAGATION OF ELECTROMAGNETIC WAVES}

The evolution of two suitably scaled slowly varying incoherently coupled wave envelopes $E_{1}$ and $E_{2}$ in a weakly nonlinear, dispersive and diffractive medium is governed by the following equations 13

$$
\begin{aligned}
& i \partial_{z} E_{1}+\alpha_{1} \vec{\nabla}_{\perp}^{2} E_{1}+\gamma_{1} \partial_{t}^{2} E_{1}+\left(\left|E_{1}\right|^{2}+\beta\left|E_{2}\right|^{2}\right) E_{1}=0, \\
& i \partial_{z} E_{2}+\alpha_{2} \vec{\nabla}_{\perp}^{2} E_{2}+\gamma_{2} \partial_{t}^{2} E_{2}+\left(\left|E_{2}\right|^{2}+\beta\left|E_{1}\right|^{2}\right) E_{2}=0,
\end{aligned}
$$

where $\vec{\nabla}_{\perp}=\vec{i} \partial_{x}+\vec{j} \partial_{y}$. Longitudinal $(z)$ and transverse $(x, y)$ coordinates are respectively measured in units of a suitable diffraction length $l_{d i f}$ and of a characteristic transverse size of the envelope. The coordinate $t$ is the retarded time scaled to the parameter $T \sqrt{l_{d i f} / l_{d i s}}$, where $T$ is the temporal duration of the envelope and $l_{d i s}$ is a characteristic GVD length. Diffraction parameters $\alpha_{1,2}$ are positive while GVD parameters $\gamma_{1,2}$ can have either sign. Rescaling $x, y, t$ once more one can always choose $\alpha_{1} / \alpha_{2}$ and $\left|\gamma_{1}\right| /\left|\gamma_{2}\right|$ to be any convenient constants. The parameter $\beta$ measures the relative strength of cross-phase modulation compare to self-phase modulation. The nonlinearity was chosen to be self-focusing because below we are interested in the dynamics of bright solitary waves.

Eqs. (1) describe a variety of physical situations but we will focus here on their application to propagation of electro-magnetic $(\mathrm{e} / \mathrm{m})$ waves. Using a circular polarization basis to describe propagation of quasimonochromatic e/m waves in isotropic dielectric materials leads to Eqs. (1), where, in such a case, $E_{1}$ and $E_{2}$ are envelopes of the left- and right- polarized components [24].
The diffraction and GVD parameters can be taken as $\alpha_{1,2}=0.5, \gamma_{1,2}=\gamma= \pm 0.5$, and $\beta=1+\chi_{x y y x}^{(3)} / \chi_{x x y y}^{(3)}$ [24], where $\chi_{i j k l}^{(3)}$ is the nonlinear susceptibility tensor. For example, $\beta=2$ for the nonresonant electronic nonlinearity and $\beta=7$ for the nonlinearity due to molecular orientation 24]. For e $/ \mathrm{m}$ waves propagating in an isotropic plasma, $\beta$ strongly depends on the ratio between the frequency of the $\mathrm{e} / \mathrm{m}$ wave and the characteristic electron plasma frequency, and it can have either sign [15]. Eqs. (1) can also be applied to describe interaction of circularly polarised waves in waveguides filled with linearly isotropic material, as, e.g., $C S_{2}$ liquid [25], in opposite situation linear coupling between $E_{1}$ and $E_{2}$ should be incorporated in the model.

Counterpropagation of scalar waves in Kerr media obeys Eqs. (1) with $\beta$ determined by the wavelengthscale refractive index gratings written by the interference pattern [17. The value of $\beta$ in this situation is directly linked with diffusion which washes out the grating making $1 \leq \beta \leq 2$ ( $\beta=2$ for zero diffusion). Envelopes of incoherent copropagating waves in Kerr media also obey Eqs. (1) with $\beta=2$ [16]. In these two situations the group velocity difference of the wave envelopes, which is not explicitly written in Eqs. (1), can be removed by a suitable phase shift.

The limiting case $\beta \rightarrow+\infty$ describes a situation with zero self-modulation effects. This approximates the so called cascading limit of non-degenerate three-wave mixing in the quadratically nonlinear media [26]. Therefore one can expect that for the large enough $\beta$ MI of the solitary solutions of Eqs. (1) should be equivalent to the MI of the three-wave solitons [23] but that it should well be different for the relatively small $\beta$.

Because the discussion of a wide range of $\beta$ values is more realistic in the context of the interaction of the circularly polarized waves, below we mainly use terminology which is appropriate to this case.

\section{MODULATIONAL INSTABILITY OF SOLITONS. GENERAL FORMULATION OF THE PROBLEM}

The primary target of the present paper is understanding of the instabilities of the ground-state, i.e. nodeless, spatially localised solutions of Eqs. (1) under the action of the $t$-dependent perturbations. These solutions are well known, see, e.g. 27 33 and refs. therein. Here, we restrict ourselves to the situation when the solitary waves are stable for $\partial_{t}=0$. Therefore we choose $\beta>0$, because it ensures absence of the 'splitting' instability [30], and $\vec{\nabla}_{\perp}=\vec{i} \partial_{x}$, to avoid collapse [7, 8 , 34].

It is important for the following to summarize relevant symmetry properties of Eqs. (1) with suppressed time derivatives $\left(\partial_{t}=0\right)$. Invariance with respect to the twoparameter gauge transformation

$$
\left(E_{1}, E_{2}\right) \rightarrow\left(E_{1} e^{i \phi_{1}}, E_{2} e^{i \phi_{2}}\right),
$$


leads to conservation of the energies $Q_{1,2}=\int d x\left|E_{1,2}\right|^{2}$ or their equivalent combinations. There are also invariances with respect to transverse translation and Galilean transformation,

$$
\begin{aligned}
& E_{1,2}(x) \rightarrow E_{1,2}\left(x+x_{0}\right), \\
& E_{1,2}(x) \rightarrow E_{1,2}(x-v z) e^{i v(x-v z / 2) .}
\end{aligned}
$$

$\phi_{1}, \phi_{2}, x_{0}$ and $v$ are free parameters. Although we use below the fact of the presence of the translational and Galilean symmetries, we do not need explicit expressions for the associated integrals of motion, which are linear momentum and 'center of mass', see, e.g., [9].

Symmetry property (2) indicates that the solitary solutions can be presented in the form

$$
E_{1,2}(x, z)=A_{1,2}(x) e^{i \kappa_{1,2} z} .
$$

$A_{1,2}(x)$ are real functions obeying the system of ordinary differential equations

$$
\frac{1}{2} \partial_{x}^{2} A_{1,2}=\kappa_{1,2} A_{1,2}-\left(A_{1,2}^{2}+\beta A_{2,1}^{2}\right) A_{1,2} .
$$

Exponential localization of the solitons requires $\kappa_{1,2}>0$. Actually one of these parameters can always be scaled away, which means that fixing one of them and varying the other in the whole region of the solitary wave existence one will capture all possible situations. However, for convenience of analytical calculations it is better to keep them both.

To study MI we seek solutions of Eq. (1) in the form of spatial solitons weakly modulated in time at frequency $\Omega \geq 0$

$$
\begin{aligned}
& E_{1,2}(x, z)=\left(A_{1,2}(x)+\right. \\
& \left.\left(U_{1,2}(x, z)+i W_{1,2}(x, z)\right) \cos \Omega t\right) e^{i \kappa_{1,2} z+i \phi_{1,2}} .
\end{aligned}
$$

Presenting solution of the linearized real system for the small perturbations $U_{m}, W_{m}$ in the form $U_{m} \sim u_{m}(x) e^{\lambda z}$ and $W_{m} \sim w_{m}(x) e^{\lambda z}$ we obtain the following eigenvalue problem (EVP)

$$
\begin{aligned}
& \left(\hat{\mathcal{L}}_{1}+\gamma \Omega^{2} \hat{I}\right) \vec{u}=-\lambda \vec{w}, \\
& \left(\hat{\mathcal{L}}_{0}+\gamma \Omega^{2} \hat{I}\right) \vec{w}=\lambda \vec{u},
\end{aligned}
$$

where $\vec{u}=\left(u_{1}, u_{2}\right)^{T}, \vec{w}=\left(w_{1}, w_{2}\right)^{T}$ and $\hat{I}$ is the identity operator. $\hat{\mathcal{L}}_{0}$ and $\hat{\mathcal{L}}_{1}$ are:

$$
\begin{gathered}
\hat{\mathcal{L}}_{0}=\left(\begin{array}{cc}
\hat{\mathcal{D}}_{1} & 0 \\
0 & \hat{\mathcal{D}}_{2}
\end{array}\right), \\
\hat{\mathcal{L}}_{1}=\left(\begin{array}{cc}
\hat{\mathcal{B}}_{1} & -2 \beta A_{1} A_{2} \\
-2 \beta A_{1} A_{2} & \hat{\mathcal{B}}_{2}
\end{array}\right),
\end{gathered}
$$

where $\hat{\mathcal{D}}_{1,2}=-\frac{1}{2} \partial_{x}^{2}+\kappa_{1,2}-A_{1,2}^{2}-\beta A_{2,1}^{2}$ and $\hat{\mathcal{B}}_{1,2}=$ $-\frac{1}{2} \partial_{x}^{2}+\kappa_{1,2}-3 A_{1,2}^{2}-\beta A_{2,1}^{2}$.
By means of simple transformation one can reduce EVP (8) to the following two EVPs for real and imaginary parts of the perturbations:

$$
\begin{aligned}
& \left(\hat{\mathcal{L}}_{0}+\gamma \Omega^{2} \hat{I}\right)\left(\hat{\mathcal{L}}_{1}+\gamma \Omega^{2} \hat{I}\right) \vec{u}=-\lambda^{2} \vec{u}, \\
& \left(\hat{\mathcal{L}}_{1}+\gamma \Omega^{2} \hat{I}\right)\left(\hat{\mathcal{L}}_{0}+\gamma \Omega^{2} \hat{I}\right) \vec{w}=-\lambda^{2} \vec{w} .
\end{aligned}
$$

EVPs (11), (12) are adjoint to each other. Therefore they have identical spectra and in case of instability the imaginary and real parts of perturbations grow with the same rates. To answer stability question it is thus enough to study only one of the EVPs, and we concentrate below on the EVP (12).

Let suppose that $\left(\kappa_{1,2}+\gamma \Omega^{2}\right)=\xi_{1,2} \geq 0$. Then, generally, $\lambda^{2} \in\left(-\infty,-\lambda_{g}^{2}\right)$ is a continuous part of the spectrum with unbounded eigenfunctions, where $\lambda_{g}=\min \left(\xi_{1}, \xi_{2}\right)$. For particular cases when $\hat{\mathcal{L}}_{1}$ becomes a diagonal operator the continuum splits into two independent bands, $\left(-\infty,-\xi_{1,2}^{2}\right)$, corresponding to unboundness of $w_{1}(x)$ and $w_{2}(x)$, respectively. Eigenvalues which do not belong to the continuum constitute the discrete part of the spectrum and have bounded eigenfunctions. Stable eigenmodes with eigenvalues obeying $-\lambda_{g}^{2}<\lambda^{2}<0$, called 'gap modes'. Any other mode of the discrete spectrum, i.e. any eigenmode with $\lambda^{2}$ complex or positive, renders the soliton unstable. If $\xi_{1}<0$ and/or $\xi_{2}<0$, the gap is closed, $\lambda_{g}=0$.

The procedure which we mainly follow to study stability of different types of solitary solutions consists of three basic steps. First, using analytical and numerical analysis we identify the discrete spectrum of EVP (12) for $\Omega=0$. Second, we develop perturbation theory for the neutral eigenmodes in the low-frequency limit, $\Omega \ll 1$. Third, we numerically build continuations of all discrete eigenvalues into the region of finite positive $\Omega$. We also allow for possible splitting of discrete eigenvalues from the edge of the continuum, but this was never actually observed.

\section{INSTABILITIES OF CIRCULARLY POLARIZED AND MANAKOV SOLITONS}

The single wave solitons of Eqs. (6) corresponding to right and left circular polarized $\mathrm{e} / \mathrm{m}$ waves are

$$
\begin{aligned}
& A_{1}(x)=\sqrt{2 \kappa_{1}} \operatorname{sech} \sqrt{2 \kappa_{1}} x, \quad A_{2}=0, \\
& A_{1}=0, \quad A_{2}(x)=\sqrt{2 \kappa_{2}} \operatorname{sech} \sqrt{2 \kappa_{2}} x .
\end{aligned}
$$

For these solutions, EVP (12), separates into two independent scalar problems. Considering for example stability of soliton with $A_{1} \neq 0$ we get:

$$
\begin{aligned}
& \left(\hat{\mathcal{N}}_{1}+\gamma \Omega^{2}\right)\left(\hat{\mathcal{N}}_{0}+\gamma \Omega^{2}\right) w_{1}=-\lambda^{2} w_{1}, \\
& \left(-\frac{1}{2} \partial_{x}^{2}+\kappa_{2}+\gamma \Omega^{2}-\beta A_{1}^{2}\right)^{2} w_{2}=-\lambda^{2} w_{2},
\end{aligned}
$$

where $\hat{\mathcal{N}}_{0}=-\frac{1}{2} \partial_{x}^{2}+\kappa_{1}-A_{1}^{2}, \hat{\mathcal{N}}_{1}=-\frac{1}{2} \partial_{x}^{2}+\kappa_{1}-3 A_{1}^{2}$. 
The operator on left-hand side of (16) has a nonnegative spectrum therefore corresponding values of $\lambda_{n}^{2}$ $(n=0,1,2,3 \ldots)$ must be nonpositive, which means absence of the unstable eigenmodes. In fact eigenvalue problem (16) can be solved analytically, see, e.g. [28,35]. The eigenvalues are

$$
\lambda_{n}^{2}=-\left(\kappa_{2}+\gamma \Omega^{2}-\frac{\kappa_{1}}{4}[\sqrt{8 \beta+1}-2 n-1]^{2}\right)^{2} .
$$

Eq. 15 is exactly an EVP arising in theory of MI of solitons in single NLS equation and details of its analytical and numerical investigations can be found in [6,8,21. For the sake of completeness and comparison with MI of the other types of solutions we summarize the main results here.

The discrete spectrum of operator $\hat{\mathcal{N}}_{1} \hat{\mathcal{N}}_{0}$ consists of two neutral eigenmodes which can be readily identified by applying infinitesimal phase and Galilean transforms to solitary wave solution. These modes are $w_{1 \phi_{1}}=A_{1}$ and $w_{1 v}=x A_{1}$. Infinitesimal translations and variations of $\kappa_{1}$ generate two neutrally stable modes of the adjoint operator $\hat{\mathcal{N}}_{0} \hat{\mathcal{N}}_{1}$ which are $u_{1 x}=\partial_{x} A_{1}$ and $u_{1 \kappa_{1}}=\partial_{\kappa_{1}} A_{1}$. These modes obey the identities: $\hat{\mathcal{N}}_{0} w_{1 \phi_{1}}=0, \hat{\mathcal{N}}_{0} w_{1 v}=$ $-u_{1 x}, \hat{\mathcal{N}}_{1} u_{1 \kappa}=-w_{1 \phi_{1}}, \hat{\mathcal{N}}_{1} u_{1 x}=0$.

Following [21] we assume $\Omega^{2} \ll 1$ and substitute the asymptotic expansions

$$
w_{1}=\left(w_{1}^{(0)}+\Omega^{2} w_{1}^{(1)}+\ldots\right)
$$

and

$$
\lambda^{2}=\Omega^{2}\left(\lambda^{(1)^{2}}+\Omega^{2} \lambda^{(2)^{2}}+\ldots\right)
$$

into Eq. (15). In the first two orders we have $\hat{\mathcal{N}}_{1} \hat{\mathcal{N}}_{0} w^{(0)}=0$ and $\hat{\mathcal{N}}_{1} \hat{\mathcal{N}}_{0} w^{(1)}=-\lambda^{(1)^{2}} w^{(0)}-\gamma\left(\hat{\mathcal{N}}_{0}+\right.$ $\left.\hat{\mathcal{N}}_{1}\right) w^{(0)}$. Solution in leading order is $w_{1}^{(0)}=C_{\phi_{1}} w_{1 \phi_{1}}+$ $C_{v} w_{1 v}$, where $C_{\phi_{1}}, C_{v}$ are constants. Orthogonality properties $\left\langle w_{1 \phi_{1}}, u_{1 x}\right\rangle=\left\langle w_{1 v}, u_{1 \kappa_{1}}\right\rangle=0$ (here and below $\left.\langle\vec{f}, \vec{g}\rangle=\sum_{m} \int d x f_{m} g_{m}\right)$ result in the independence of the branches produced by the phase and Galilean neutral modes. Therefore $C_{\phi_{1}}$ and $C_{v}$ are in fact independent constants. Solvability condition of the 1st order problem gives

$$
\begin{aligned}
\lambda_{\phi_{1}}^{(1)^{2}} & =2 \gamma \frac{Q_{1}}{\partial_{\kappa_{1}} Q_{1}}=4 \gamma \kappa_{1}, \\
\lambda_{v}^{(1)^{2}} & =-2 \gamma \frac{\left\langle u_{1 x}, u_{1 x}\right\rangle}{Q_{1}}=-\frac{4}{3} \gamma \kappa_{1} .
\end{aligned}
$$

Eqs. (20), (21) indicate onset of instability for either sign of $\gamma$. However, the character of the instability depends on the sign of $\gamma$. For anomalous GVD $(\gamma>0)$ the spatially symmetric eigenmode becomes unstable leading to clustering of the soliton stripe into filaments (neck MI). For normal GVD $(\gamma<0)$ an excitation of the antisymmetric eigenmode leads to spatial symmetry breaking and bending of the solitary stripe along the temporal coordinate (snake MI). The period of the modulations is approximately equal to $2 \pi / \Omega_{\max }$, where $\Omega_{\max }$ is the maximally unstable frequency.

Typical dependencies of the MI growth rates vs $\Omega$ are presented at Fig. 1. The neck instability disappears at $\Omega_{\phi_{1}}=\sqrt{3 \kappa_{1} / \gamma}$ where $w_{1}=0$ and $u_{1}=\operatorname{sech}^{2} \sqrt{2 \kappa_{1}} x$. For $\Omega>\Omega_{\phi_{1}}$ the corresponding eigenmode becomes a gap mode. Note that for $\gamma>0$ the gap becomes wider with increasing of $\Omega$. The snake instability disappearance is very difficult to track numerically because the corresponding eigenmode develops oscillating tails and becomes weakly localized, so that a larger number of the grid points is required. However, our numerical analysis clearly indicates that the branch of snake MI does not disappear stepwise at the point where the gap is closed, $\Omega_{g}=\sqrt{-\kappa_{1} / \gamma}$, as was suggested in Ref. [6], but continues beyond this point and probably reaches $\lambda^{2}=0$ at some larger $\Omega$.

The nonlinear stage of MI is also perfectly analogous to that in the single NLS. Filaments, formed as the result of the neck MI development, collapse to a singularity during further propagation [7, 8$]$. The snake MI leads to soliton spreading due to unbalanced action of the self-focusing nonlinearity and normal GVD [8]. The second field $E_{2}$ is not affected by the discussed instabilities, because of the incoherent nature of the coupling between $E_{1}$ and $E_{2}$.

In the special case $\beta=1, \alpha_{1}=\alpha_{2}$ and $\gamma_{1}=\gamma_{2}$ Eqs.(1) are invariant under the arbitrary rotations in $\left(E_{1}, E_{2}\right)$ plane, $E_{1,2} \rightarrow \cos \vartheta E_{1,2} \pm \sin \vartheta E_{2,1}$. This leads to a new parameterization of the ground state solitons. These are usually called Manakov solitons [36] and they are given by the solutions of Eqs. (6) with $\kappa_{1,2}=\kappa$ :

$$
A_{1}=\cos \theta A(x), \quad A_{2}=\sin \theta A(x)
$$

Here the angle $\theta$ is a new free parameter characterizing the polarization angle, and $A(x)=\sqrt{2 \kappa} \operatorname{sech} \sqrt{2 \kappa} x$. Because of the rotational invariance, Manakov solitons with different polarizations are equivalent and their MI is indepent of the polarization angle. Therefore one can always set $\theta=0$, and then the corresponding EVP coincides with Eqs. (15), 16.

\section{INSTABILITIES OF LINEARLY AND ELLIPTICALLY POLARIZED SOLITONS $(\beta \neq 1)$}

\section{A. Soliton family and associated neutral modes}

To study solitons of an arbitrarily polarization for $\beta \neq 1$, i.e. $A_{1} \neq 0$ and $A_{2} \neq 0$, it is more convenient to introduce absolute, $\varphi=\frac{1}{2}\left(\phi_{1}+\phi_{2}\right)$, and relative, $\psi=\frac{1}{2}\left(\phi_{1}-\phi_{2}\right)$, phases. The corresponding integrals of motion are the total energy $Q=Q_{1}+Q_{2}$ and energy unbalance $Q_{u}=Q_{1}-Q_{2}$. Associated soliton parameters are $\kappa=\frac{1}{2}\left(\kappa_{1}+\kappa_{2}\right)$ and $\delta=\frac{1}{2}\left(\kappa_{1}-\kappa_{2}\right)$.

For $\delta=0$ there is an obvious and well known analytical solution of Eqs. (6) 9,28 33. 


$$
A_{1,2}(x)=A(x)=\sqrt{\frac{2 \kappa}{1+\beta}} \operatorname{sech} \sqrt{2 \kappa} x,
$$

corresponding to a linearly polarized soliton. Using numerical solution of Eq. (16) one can verify that for $\beta \neq 1$ the exact solution (23) belongs to the family of the solitary solutions parameterized by $\kappa$ and $\delta$.

Using Eq. (17) for $n=0, \Omega=0$ and its analog for the solution (14) we conclude that for fixed values of $\kappa$ and $\beta$, a family of ground state coupled solitary solutions of Eqs. (6) exists for $\delta \in\left(-\delta_{c}, \delta_{c}\right)$, where

$$
\delta_{c}=\kappa\left|\frac{1-4 \beta+\sqrt{1+8 \beta}}{3+4 \beta-\sqrt{1+8 \beta}}\right| .
$$

Analogs of Eqs. (24) have been derived before in a number of papers using different methods, see, e.g. [9, 27, 32]. Expression under the modulus in Eq. (24) changes its sign from plus to minus once $\beta$ changes from $\beta<1$ to $\beta>1$. It follows that for $\beta<1$ the family of the elliptically polarized solitons splits from the family $A_{2}=0$ $\left(A_{1}=0\right)$ of the circularly polarized ones at $\delta=\delta_{c}$ $\left(\delta=-\delta_{c}\right)$ and this is vice versa for $\beta>1$. Continuous variation of $\delta$ from $-\delta_{c}$ to $\delta_{c}$ for fixed $\kappa$ and $\beta<1(\beta>1)$ results in monotonic decay of $Q_{2}\left(Q_{1}\right)$ from its maximal value $Q_{+}\left(Q_{-}\right)$down to zero and in growth of $Q_{1}$ $\left(Q_{2}\right)$ from zero upto $Q_{+}\left(Q_{-}\right)$, where $Q_{ \pm}=2 \sqrt{2\left(\kappa \pm \delta_{c}\right)}$. Therefore, we can make an important for the following conclusion, that for $\beta<1 \partial_{\delta} Q_{u}>0$ and for $\beta>1$ $\partial_{\delta} Q_{u}<0$. Numerically build dependencies of $Q_{u}$ vs $\delta$ for different values of $\beta$ are presented in Fig. 22.

Consider now the main spectral properties of the elliptically polarized solitons for $\Omega=0$ and $\beta \neq 0,1$. Phase and Galilean symmetries generate three neutral eigenmodes of the EVP (12), they are $\vec{w}_{\varphi}=\left(A_{1}, A_{2}\right)^{T}$, $\vec{w}_{\psi}=\left(A_{1},-A_{2}\right)^{T}$, and $\vec{w}_{v}=x\left(A_{1}, A_{2}\right)^{T}$. Infinitesimal variations of $\kappa$ and $\delta$, and translational symmetry generate neutral modes of the adjoint problem (11): $\vec{u}_{\kappa}=$ $\partial_{\kappa}\left(A_{1}, A_{2}\right)^{T}, \vec{u}_{\delta}=\partial_{\delta}\left(A_{1}, A_{2}\right)^{T}$, and $\vec{u}_{x}=\partial_{x}\left(A_{1}, A_{2}\right)^{T}$. These six modes obey the following identities $\hat{\mathcal{L}}_{0} \vec{w}_{\varphi}=0$, $\hat{\mathcal{L}}_{0} \vec{w}_{\psi}=0, \hat{\mathcal{L}}_{0} \vec{w}_{v}=-\vec{u}_{x}, \hat{\mathcal{L}}_{1} \vec{u}_{\kappa}=-\vec{w}_{\varphi}, \hat{\mathcal{L}}_{1} \vec{u}_{\delta}=-\vec{w}_{\psi}$, $\hat{\mathcal{L}}_{1} \vec{u}_{x}=0$.

For $\beta=0$ Eqs. (1) separate in two independent NLS equations. The independence of the two fields results in additional translational and Galilean symmetries characterizing freedom of the relative transverse translation and motion of the two waves. Therefore EVPs (11) and (12) have additional neutral modes $\vec{u}_{\delta x}=\partial_{x}\left(A_{1},-A_{2}\right)^{T}$, $\vec{w}_{\delta v}=x\left(A_{1},-A_{2}\right)^{T}$. As numerical solution for $0<\beta<$ 1 shows the corresponding eigenvalue produces stable branch of the discrete spectrum. For $|\beta| \ll 1$ approximate expression for this eigenvalue can be readily found [29,30, $\lambda_{\delta v}^{2}=-64 \beta / 15$. Excitation of the corresponding eigenmode results in position oscillations of the soliton upon its propagation 31]. When $\beta \rightarrow 1$ this eigenmode disappears into the continuum 31.

\section{B. Asymptotic stability analysis $\left(\Omega^{2} \ll 1\right)$}

Now assuming that $\beta \gg \Omega^{2}$ we can use the asymptotic techniques described in the previous section to continue zero-eigenvalue modes into the region $\Omega^{2} \ll 1$. Making substitutions

$$
\vec{w}=\left(\vec{w}^{(0)}+\Omega^{2} \vec{w}^{(1)}+\ldots\right)
$$

and of Eq. 119) into (12) we get in the first two orders: $\quad \hat{\mathcal{L}}_{1} \hat{\mathcal{L}}_{0} \vec{w}^{(0)}=0$ and $\hat{\mathcal{L}}_{1} \hat{\mathcal{L}}_{0} \vec{w}^{(1)}=-\lambda^{(1)^{2}} \vec{w}^{(0)}-$ $\gamma\left(\hat{\mathcal{L}}_{0}+\hat{\mathcal{L}}_{1}\right) \vec{w}^{(0)}$. Solution in the leading order is $\vec{w}^{(0)}=$ $C_{\varphi} \vec{w}_{\varphi}+C_{\delta} \vec{w}_{\delta}+C_{v} \vec{w}_{v}$. As in the previous subsection one can show that the branches produced by the two phase modes on the one hand and by the Galilean mode on the other are independent. The solvability condition of the first order problem for the Galilean mode gives

$$
\lambda_{v}^{(1)^{2}}=-2 \gamma \frac{\left\langle\vec{u}_{x}, \vec{u}_{x}\right\rangle}{Q},
$$

which implies snake instability for $\gamma<0$. When $\delta=0$, $\lambda_{v}^{(1)^{2}}=-4 \gamma \kappa / 3$, cf. Eq. (21).

For the two phase modes the solvability condition results in a quadratic equation for $\lambda^{(1)^{2}}$

$$
a \lambda^{(1)^{4}}+b \lambda^{(1)^{2}}+c=0,
$$

where

$$
\begin{gathered}
4 a=\partial_{\kappa} Q_{u} \partial_{\delta} Q-\partial_{\kappa} Q \partial_{\delta} Q_{u}, \\
2 b=\gamma Q\left(\partial_{\kappa} Q+\partial_{\delta} Q_{u}\right)-\gamma Q_{u}\left(\partial_{\kappa} Q_{u}+\partial_{\delta} Q\right), \\
c=-4 \gamma^{2} Q_{1} Q_{2} .
\end{gathered}
$$

Corresponding values of $C_{\varphi}$ and $C_{\psi}$ are linked through the equality

$$
\frac{C_{\varphi}}{C_{\psi}}=\frac{2 \gamma Q-\lambda^{(1)^{2}} \partial_{\delta} Q_{u}}{\lambda^{(1)^{2}} \partial_{\delta} Q-2 \gamma Q_{u}}
$$

where $\lambda^{(1)^{2}}$ is the corresponding root of Eq. (27). In the general case expressions for the roots of Eq. (27) can not be analyzed analytically, but it is already clear that four symmetric neck type eigenmodes exist and either two or all of them may be responsible for instability.

If $\delta=0$ then $\left\langle\vec{w}_{\varphi}, \vec{u}_{\delta}\right\rangle=\left\langle\vec{w}_{\psi}, \vec{u}_{\kappa}\right\rangle=0$ and therefore $\vec{w}_{\varphi}$ and $\vec{w}_{\psi}$ eigenmodes produce independent branches of the discrete spectrum. This results in independence between $C_{\varphi}$ and $C_{\psi}$ and simplifies formulas for the associated eigenvalues:

$$
\begin{aligned}
\lambda_{\varphi}^{(1)^{2}} & =2 \gamma \frac{Q}{\partial_{\kappa} Q}=4 \gamma \kappa, \\
\lambda_{\psi}^{(1)^{2}} & =2 \gamma \frac{Q}{\partial_{\delta} Q_{u}}=2 \gamma \kappa f(\beta) .
\end{aligned}
$$


Here $f(\beta)=\left(\int \operatorname{sech} x g(x, \beta) d x\right)^{-1}$ and function $g$ obeys to $\left(\partial_{x}^{2}-1+2 \frac{3-\beta}{1+\beta} \operatorname{sech}^{2} x\right) g=\operatorname{sech} x . \quad f(\beta)$ changes its sign from plus to minus when $\beta$ passes through unity, see Fig. 3. Alternatively, Eq. (29) can be rewritten as $\lambda_{\psi}^{(1)^{2}}=2 \gamma Q_{1} / \partial_{\delta} Q_{1}$, cf. Eq. (4) in [23].

The $\lambda_{\varphi}^{(1)^{2}}$ eigenvalue and associated neutral mode $\vec{w}_{\varphi}$ are linked to the symmetry in the absolute phase $\varphi$ and have their analogies in the spectral problem for single wave solitons described in the previous section, see Eq. (20). The $\lambda_{\psi}^{(1)^{2}}$ eigenvalue and neutral mode $\vec{w}_{\psi}$ are novel. They can be directly attributed to the symmetry in the differential phase $\psi$. This branch of the discrete spectrum generates instability for normal GVD $(\gamma<0)$ if $\beta>1$ and for anomalous GVD if $\beta<1$, see Figs. 4, 6. Thus, the asymptotic analysis indicates that for $\beta>1, \gamma<0$ neck and snake instabilities coexist, and for $\beta<1, \gamma>0$ two different types of neck instability coexist. Numerical evaluation of the roots of Eq. (27) shows that the same conclusions hold also for $\delta \neq 0$, throughout the whole existence region of the family of elliptically polarized solitons. Solving the EVP (12) numerically, we find that in the low-frequency limit the instability growth rates match those predicted by our perturbation theory within of few percent up to $\Omega \simeq 0.5$. Numerical investigation (for more details see below) also shows that apart from the three instabilities discussed in previous subsection, a fourth MI associated with continuation of $\vec{w}_{\delta v}$ into the region of $\beta \neq 0, \Omega \neq 0$ also exists. Analytical treatment of this instability is also possible, but it will not be pursued here, because corresponding MI branch is never dominant.

Let us first discuss in general terms the physical meaning of all the different types of the instabilities in the simple situation with zero imbalancing $(\delta=0)$, and only then we will proceed with details of the numerical analysis.

\section{Instability induced polarization dynamics}

All eigenmodes of the EVP 12 are two component vectors, whose first and second components are responsible for the spatial form of modulations of the fields $E_{1}$ and $E_{2}$, respectively. The eigenmodes $\vec{w}_{\varphi}$ and $\vec{w}_{v}$ corresponding to the variations of the absolute phase $\varphi$ and of the absolute velocity $v$ of the coupled solitons have first and second components which are in phase for any value of $x$. This property holds throughout the whole region of existence of the associated branches of the discrete spectrum. Therefore an excitation of these eigenmodes is not accompanied by the breaking of the polarization of the initial state. In contrast, the eigenmodes linked with $\vec{w}_{\psi}$ and $\vec{w}_{\delta v}$ neutral modes, or in other words with variations of the relative phase $\psi$ and the relative velocity $\delta v$, have anti-phased first and second components. Therefore their excitation does lead to polarization symmetry breaking. In particular, one should expect that destabilization of the eigenmode associated with the relative phase $\psi$ will result in breaking of the linearly polarized soliton stripe into a chain of circularly polarized clusters, where neighboring clusters have opposite (left and right) polarizations. The same conclusions are obviously valid for the real parts of the perturbations.

Note here, that by direct substitution of the linearly polarized solution Eq. (23) into the EVPs (11), (12) one can easily show that the eigenvalues of the eigenmodes with in-phase first and second components are independent of $\beta$. This means physically that in-phase MI is insensitive to the relative strength of self- and cross-phase modulations.

\section{Numerical results for normal GVD $(\gamma<0)$}

We start description of our numerical results with discussion of the normal GVD case. We found two snake instabilities for $\beta<1$, see Fig. 4. One of them corresponds to the in-phase snaking of both fields, see Fig. 4(c), and its growth rate in the low frequency limit is given by the Eq. (26). The other one corresponds to the anti-phase snaking, see Figs. 4(d). Examples of the growth rate dependencies vs $\Omega$ and details of the anti-phase snaking appearance are presented in Fig. 4(a) and Fig. 5, respectively. Dependencies of the maximal instability growth rates on $\beta$ are presented in Fig. A(b). We found that inphase snaking dominates anti-phase snaking for all values of $\delta$ and $\beta$. For $\beta=1$ the anti-phase snake mode disappears inside the continuum and does not appear again for all $\beta>1$.

The dominant role of the in-phase snake instability means that breaking of the polarization state imposed by the initial conditions is unlikely to happen upon propagation. Introducing imbalancing for a fixed total energy enhances this dominance, see Fig. 4(a). Thus, when $\beta<1$, the linearly polarized solitons are more stable than any other state of polarization.

The anti-phase neck MI associated with the relative phase $\psi$ appears for $\beta>1$, see Eq. (29) and Figs. 6(b). The in-phase snake instability obviously also exists, see Eq. (26) and Figs. 6(c),(d). The in-phase snake MI dominates the anti-phase neck for $1<\beta<\beta_{s n}$ and vice versa for $\beta>\beta_{s n}$, see Fig. 7 , where the cross-over value $\beta_{s n}$ depends weakly on $\delta$. This fact can also be seen from the comparison of the perturbative results for $\delta=0$. According to the Eqs. (28), (29) and Fig. (7) the neck instability dominates the snake in the low frequency limit starting from $\beta \simeq 3.55$. Numerical stability analysis gives that $\beta_{s n} \simeq 3.47$ at $\Omega=\Omega_{\max }$ for $\delta=0$. Introducing imbalancing always leads to the suppression of the both instabilities, see Figs. 6(a),(c) and Fig. 7. Therefore the circular polarized soliton is most stable for a given energy.

In analogy with MI of circularly polarized solitons for 
normal GVD, the neck and snake unstable eigenmodes become weakly confined and develop oscillating tails as $\Omega$ increases beyond the point where the gap is closed, $\lambda_{g}=0$.

To test our linear stability analysis and study the nonlinear evolution we performed a series of computer simulations of the Eqs. (1) with initial conditions in the form of a soliton stripe perturbed by spatio-temporal white noise of order of few percent. Typical simulation results are presented in Fig. 8,9,10. For $\beta<\beta_{s n}$ we observed in-phase snaking of the stripe along the temporal dimension, see Fig. 8. For $\beta>\beta_{\text {sn }}$ the soliton stripe breaks in such a way as to form the interleaved intensity peaks of $E_{1}$ and $E_{2}$, see Fig. 9, as expected when the out of phase neck MI is dominant. The spatio-temporal patterns formed at the initial stage of MI finally spread because of the unbalanced action of the normal GVD and self-focusing nonlinearity. For $\beta \simeq \beta_{\text {sn }}$ we observed competition between the neck and snake MIs, see Fig. 10. In Fig. $10\left(b_{1}\right),\left(b_{2}\right)$ one can clearly see that at the intermediate stage of MI the typical in-phase snake pattern is superimposed on the anti-phase neck pattern.

Thus, we conclude, that in the media with normal GVD spatial soliton stripes perfectly develop snake MI without polarization symmetry breaking if $\beta \in\left(0, \beta_{s n}\right)$ and neck MI with polarization symmetry breaking if $\beta \in\left(\beta_{\text {sn }},+\infty\right)$.

\section{E. Numerical results for anomalous GVD $(\gamma>0)$}

There are two neck MIs in this case for $\beta<1$, see Fig. 11. One of them is associated with the absolute phase $\varphi$ and corresponds to the in-phase neck MI. The other one is associated with the relative phase $\psi$ and corresponds to the anti-phase neck MI. The in-phase MI dominates the anti-phase one for any value of $\delta$ and $\beta$, which means conservation of the polarization state imposed by the initial conditions. Nonzero imbalancing for fixed total energy leads to the growth of the in-phase MI and to the suppression of the anti-phase one, see Fig. 11(a),(b). For $\beta>1$ only in-phase instability exists, but now imbalancing leads to the suppression of the instability, see Fig. 12. Presence of these instabilities agrees with the predictions of low-frequency analysis, see Eqs. (28), (29) and Figs. 2,3. A typical result of the numerical simulation of the neck instability development is shown in Fig. 13. Note that the numerically attainable propagation distance was limited by the distance at which the most intense filaments formed at the initial stage of MI collapse to singularities.

Cut-off frequencies, where the neck MIs disappear can be found analytically for $\delta=0$. Growth rate of the inphase MI becomes zero at $\Omega=\sqrt{3 \kappa / \gamma}$ in full analogy with single NLS equation, see section IV. The anti-phase MI disappears at $\Omega^{2}=\kappa(D-B) /(2 \gamma)$ having $\vec{w}=0$ and $u_{1,2}=(\operatorname{sech} \sqrt{2 \kappa} x)^{(B-1) / 2}$, here $D=(11-5 \beta) /(1+\beta)$,
$B=\sqrt{(25-7 \beta) /(1+\beta)}$.

Thus, in the media with anomalous GVD spatial soliton stripe always develops neck MI without polarization symmetry breaking and filamentary structure formed during this process collapses upon propagation. Detailed study of collapse in coupled NLS equations is outwith the scope of this paper. Some details on this issue can be found in 34.

\section{DISCUSSION}

It is interesting to compare MI of solitons with results on MI of CWs 13, which can be easily recovered from Eqs. (6), (11), (12) putting $\partial_{x}^{2}=0$. For simplicity we again consider the case of the linear polarization, $E_{1,2}=\sqrt{\kappa /(1+\beta)} e^{i \kappa z}$. Then corresponding eigenvalues are $\lambda_{\varphi}^{2}=\gamma \Omega^{2}\left(2 \kappa-\gamma \Omega^{2}\right)$ and $\lambda_{\psi}^{2}=$ $\gamma \Omega^{2}\left(2 \kappa(1-\beta) /(1+\beta)-\gamma \Omega^{2}\right)$. For normal GVD, $\lambda_{\psi}^{2}$ can be positive only for $\beta>1$. For anomalous GVD, $\lambda_{\varphi}^{2}$ generates instability for any $\beta$ and $\lambda_{\psi}^{2}$ only for $\beta<1$. Thus, as one could expect, neck instabilities of solitons related to the phase symmetries have analogies for CWs. Snake instabilities are obviously absent for CWs, which is the main difference between the dynamics of spatially confined solitons and infinitely extended CWs. Namely, in the case of normal GVD, CWs are modulationally stable for $\beta<1$ and unstable for $\beta>1$ (demonstrating polarization symmetry breaking). Solitons are snake unstable in this situation for any $\beta$ and this instability does not involve changes in the polarization state. However, starting from a critical value of $\beta=\beta_{\text {sn }}$ the snake instability becomes suppressed by the neck one, which is analogous to instability of CW. This instability does lead to polarization symmetry breaking. In particular, a linearly polarized soliton breaks, due to this instability, into the chain of circularly polarized clusters. Because snake instability leads to spatial symmetry breaking and neck MI does not, the change in MI of solitons at $\beta=\beta_{\text {sn }}$ can be interpreted as a transition from spatial symmetry breaking to polarization symmetry breaking.

In the limit situation $\beta \gg 1$ self-phase effects are negligible compare to cross-phase ones and development of the in-phase and anti-phase neck MIs can be qualitatively explained using Fermat's principle. Due to MI development the effective refractive index for $E_{1}$ and $E_{2}$ fields gets modulated through the XPM mechanism with pe$\operatorname{riod} 2 \pi / \Omega_{\max }$. This results in temporal cross-defocusing of filaments in media with normal GVD and in crossfocusing for anomalous GVD. Thus, in the case of normal GVD, interleaved pattern of the intensity peaks of $E_{1}$ and $E_{2}$ fields should be preferable because it enables each field to see a refractive index that increases to its peak, i.e. one that is in accord with Fermat's principle. This is clearly verified in Fig.9. The same arguments lead to the conclusion that a pattern with all intensity peaks coincident is preferable for anomalous GVD. 
Considering possibility of experimental observations of predicted phenomena, we have to say that diffraction induced MI of soliton-like stripe, which is formally equivalent to the case of anomalous GVD, is probably easiest to observe. However, it is less interesting at the same time because it is perfectly analogous to MI of CWs and it is not accompanied by any polarization effects. More interesting dynamics is expected in media with normal GVD. In fact, experimental observation of temporal splitting induced by normal GVD of spatially confined pulses in a self-focusing medium was recently reported in [37]. However, transverse and polarization effects, which, accordingly to our results, should play an important role, were not studied during this experiment. Numerical studies 37] presented to support the experimental results were restricted by scalar approximation and radial geometry.

The rescaled instability growth rate $\lambda$ as function of the modulational frequency $\Omega$ can be related to physical units using the formulae:

$$
\lambda_{p h}=\frac{\lambda}{4 k w^{2}}, \Omega_{p h}^{2}=\frac{\gamma \Omega^{2}}{2 k k^{\prime \prime} w^{2}} .
$$

Here $\lambda_{p h}$ and $\Omega_{p h}$ are the instability growth rate and modulational frequency in physical units, $k$ is the wave vector, $w$ is the beam width, $k^{\prime \prime}=\partial_{\omega}^{2} k$. For example for radiation at $1 \mu \mathrm{m}$ propagating in an AlGaAs planar waveguide $k^{\prime \prime} \simeq-10^{-23} s^{2} / m[38]$ and for typical soliton transverse size $w \simeq 50 \mu m$ [39] we get $\lambda_{p h} \simeq \lambda /(5 \mathrm{~cm})$ and $\Omega_{p h}^{2} \simeq \Omega^{2} /\left(10^{-25} s^{2}\right)$. For experiments with fused silica at wavelength $830 \mathrm{~nm}$, see second from Refs. [37, $k^{\prime \prime} \simeq-10^{-26} s^{2} / m$ and $\Omega_{p h}^{2} \simeq \Omega^{2} /\left(10^{-28} s^{2}\right)$.

\section{SUMMARY}

We have analyzed and described dispersive MI of families of nodeless spatial solitons in the system of the two incoherently coupled NLS equations. Considering coupled soliton states, we have established the existence of the four branches of instabilities, which are linked to the symmetries in the absolute and relative phases and in the absolute and relative motions of solitons. We gave a physical interpretation of our results describing GVDinduced polarization dynamics. In particularly, we found that in media with normal GVD the MI induced spatial symmetry breaking in the transverse plane changes to the polarization symmetry breaking when the relative strength of the cross-phase modulation exceeds a certain threshold value. In media with anomalous GVD, MI results in breaking of spatial solitons into spatio-temporal clusters which collapse upon further propagation. This is not followed by either spatial or polarization symmetry breaking.
[1] G.A. Askar'yan, Zh. Eksp. Teor. Fiz. 42, 1567 (1962) [Sov. Phys. JETP 15, 1088 (1962)]; T. Taniuti and H. Washimi, Phys. Rev. Lett. 21, 209 (1968).

[2] V.I. Bespalov and V.I. Talanov, Pis'ma Zh. Eksp. Teor. Fiz. 3, 471 (1966) [JETP Lett. 3, 307 (1966)]; L.A. Ostrovskii, Zh. Eksp. Teor. Fiz. 51, 1189 (1966) [Sov. Phys. JETP 24, 797 (1967)]; V.I. Karpman, Pis'ma Zh. Eksp. Teor. Fiz. 6, 759 (1967) [JETP Lett. 6, 227 (1967)].

[3] T.B. Benjamin and J.E. Feir, J. Fluid Mech. 27, 417 (1967).

[4] N.F. Pilipetskii and A.R. Rustamov, Pis'ma Zh. Eksp. Teor. Fiz. 2, 88 (1965) [JETP Lett. 2, 55 (1965)].

[5] A comprehensive review of MI in hamiltonian systems is beyond our present scope, therefore our list of references is restricted to original works [1, 4, 22], some key reviews [6] 11] and to papers which have direct relevance to the present work.

[6] E.A. Kuznetsov, A.M. Rubenchik, and V.E. Zakharov, Phys. Rep. 142, 103 (1986).

[7] J.J. Rasmussen, K. Rypdal, Physica Scripta 33, 481 (1986).

[8] L. Berge, Phys. Rep. 303, 259 (1998).

[9] N.N. Akhmediev and A. Ankievich, Solitons: Nonlinear Pulses and Beams (Chapman \& Hall, London, 1997) and refs. therein.

[10] A.V. Mamaev, M. Saffman, D.Z. Anderson, and A.A. Zozulya, Phys. Rev. A 54, 870 (1996).

[11] Y.S. Kivshar and B. Luther-Davies, Phys. Rep. 298, 81 (1998).

[12] R.K. Dodd, J.C. Eilbeck, J.D. Gibbon, and H.C. Morris, Solitons and nonlinear wave equations (Academic Press, London, 1984).

[13] A.L. Berkhoer and V.E. Zakharov, Zh. Eksp. Teor. Fiz. 58, 903 (1970) [Sov. Phys. JETP 31, 486 (1970)].

[14] G.J. Roskes, Stud. Appl. Math. 55, 231 (1976).

[15] C.J. McKinstrie and R. Bigham, Phys. Fluids B 1, 230 (1988); G.G. Luther and C.J. McKinstrie, J. Opt. Soc. Am B 7, 1125 (1990); M. Yu, C.J. McKinstrie, and G.P. Agrawal, Phys. Rev. E 482178 (1993).

[16] G.P. Agrawal, Phys. Rev. Lett. 59, 880 (1987); J. Opt. Soc. Am B 7, 1072 (1990).

[17] W.J. Firth and C. Paré, Opt. Lett. 13, 1096 (1988); W.J. Firth, A. Fitzgerald, and C. Paré, J. Opt. Soc. Am B 7, 1087 (1990).

[18] M. Yu, C.J. McKinstrie, and G.P. Agrawal, J. Opt. Soc. Am B 15, 607 (1998).

[19] J.E. Rosenberg, Phys. Rev. A 42, R682 (1990).

[20] M. Haelterman and A.P. Sheppard, Phys. Rev. E 49, 3389 (1994).

[21] V.E. Zakharov, Zh. Eksp. Teor. Fiz. 53, 1735 (1967) [Sov. Phys. JETP 26, 994 (1968)]; V.E. Zakharov and A.M. Rubenchik, Zh. Eksp. Teor. Fiz. 65, 997 (1973) [Sov. Phys. JETP 38, 494 (1974)].

[22] B.B. Kadomtsev and V.I. Petviashvily, Dokl. Acad. Nauk SSSR 192, 753 (1970) [Sov. Phys. Dokl. 15, 539 (1970)].

[23] D.V. Skryabin and W.J. Firth, Phys. Rev. Lett. 81, 3379 (1998).

[24] R.W. Boyd, Nonlinear Optics (Academic Press, Boston, 1992).

[25] D. Wang, R. Barille, and G. Rivoire, J. Opt. Soc. Am. B 
15, 2731 (1998).

[26] H.T. Tran, Opt. Commun. 118, 581 (1995).

[27] V.M. Eleonsky, V.G. Korolev, N.E. Kulagin, and L.P. Shilnikov, Zh. Eksp. Teor. Fiz. 99, 1113 (1991) [Sov. Phys. JETP 72, 619 (1991)]

[28] B.A. Malomed, J. Opt. Soc. Am. B 9, 2075 (1992).

[29] T. Ueda and W.I. Kath, Phys. Rev. A 42, 563 (1990).

[30] J. Yang and D.J. Benney, Stud. Appl. Math. 96, 111 (1996).

[31] J. Yang, Stud. Appl. Math. 98, 61 (1997).

[32] J.K. Yang, Physica D 108, 92 (1997).

[33] D.J. Kaup, B.A. Malomed and R.S. Tasgal, Phys. Rev. E 48, 3049 (1993). B.A. Malomed and R.S. Tasgal, Phys. Rev. E 58, 2564 (1998).

[34] C.J. McKinstrie and D.A. Russel, Phys. Rev. Lett. 61, 2929 (1988); A.A. Afanas'ev, V.I. Kruglov, B.A. Samson, R. Jakyte, and V.M. Volkov, J. Mod. Opt. 38, 1189 (1991); L. Gagnon, J. Phys. A 25, 2649 (1992); T. Tsurumi and M. Wadati, J. Phys. Soc. Japan 67, 93 (1998).

[35] L.D. Landau and E.M. Lifshitz, Quantum Mechanics (Pergamon Press, Oxford, 1965).

[36] S.V. Manakov, Zh. Eksp. Teor. Fiz. 65, 505 (1973) [Sov. Phys. JETP 38, 248 (1974)].

[37] J.K. Ranka, R.W. Schirmer, and A.L. Gaeta, Phys. Rev. Lett. 77, 3783 (1996); A.A. Zozulya, S.A. Diddams, A.G. Van Engen, and T.S. Clement, Phys. Rev. Lett. 82, 1430 (1999).

[38] N. Belanger, A. Villeneuve, and J.S. Aitchison, J. Opt. Soc. Am. B 14, 3003 (1997).

[39] J.S. Aitchison, D.C. Hutchings, J.M. Arnold, J.U. Kang, G.I. Stegeman, E. Ostrovskaya, and N. Akhmediev, J. Opt. Soc. Am. B 14, 3032 (1997).

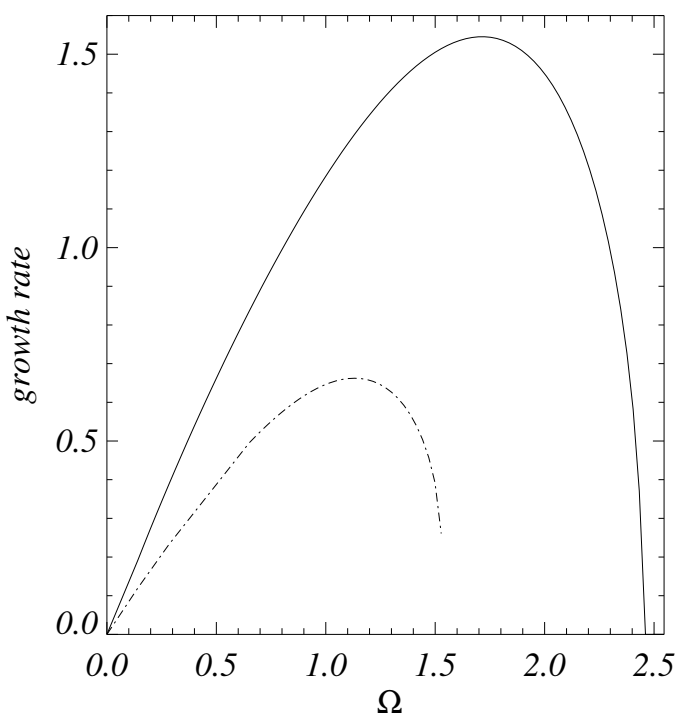

FIG. 1. Instability growth rates of the circularly polarized soliton vs $\Omega, \kappa_{1}=1$. Full (dot-dashed) line is for neck (snake) MI, $\gamma=0.5(\gamma=-0.5)$.

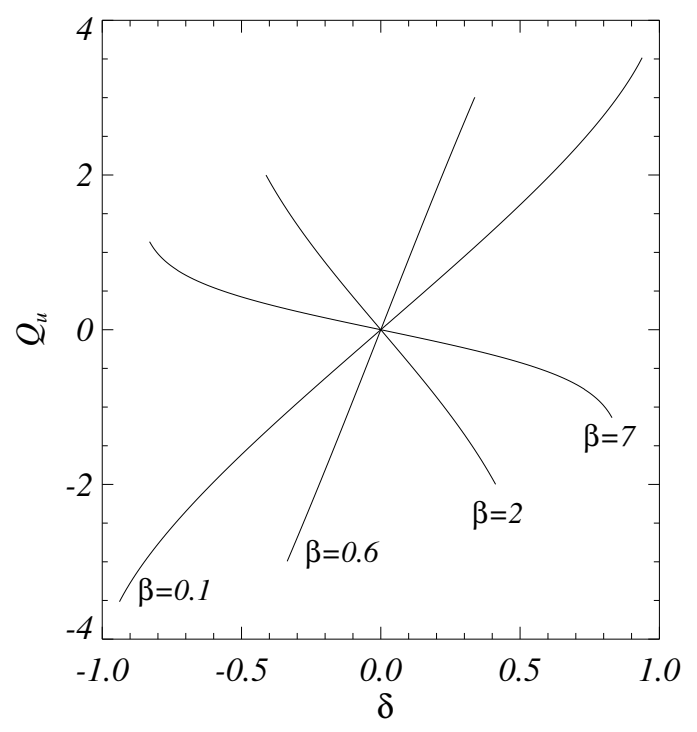

FIG. 2. Energy unbalancing $Q_{u}$ vs $\delta, \kappa=1$.

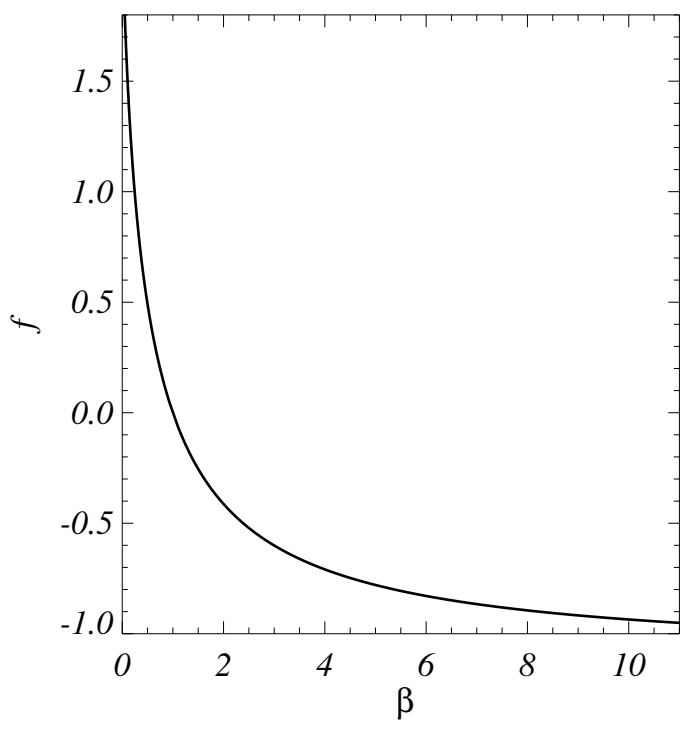

FIG. 3. Function $f(\beta)$, see Eq. (29). 

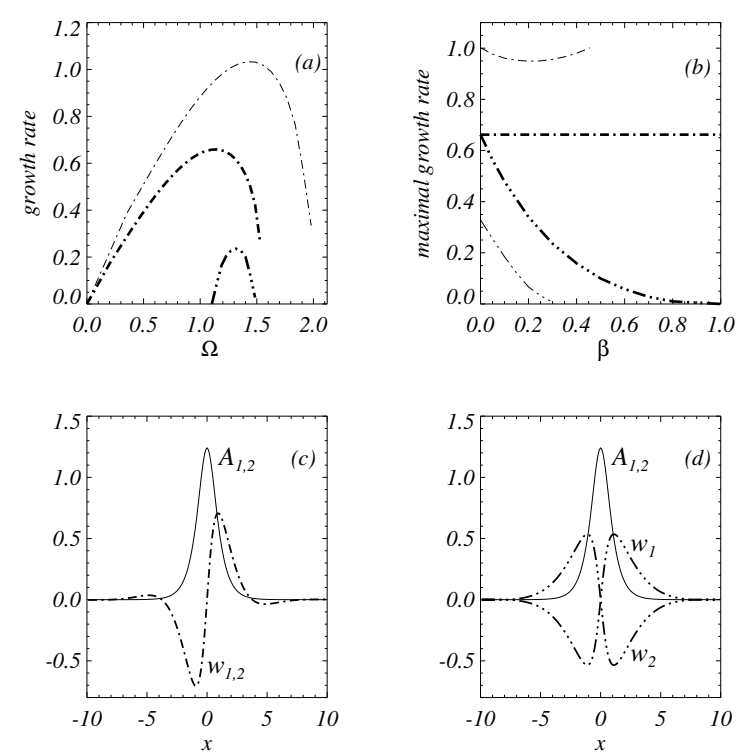

FIG. 4. Instability growth rates, spatial profiles of the solitary solutions and of the unstable eigenmodes for $\beta<1$, $\gamma=-0.5$. Dash-dot (dash-dot-dot-dot) lines correspond to the in-phase (anti-phase) snake MI. (a) Growth rates vs $\Omega$, $\beta=0.3$. Thin (thick) lines correspond to $\kappa=1, \delta=0$, $Q \simeq 4.04, Q_{u}=0\left(\kappa=1.155, \delta=0.5, Q \simeq 4.04, Q_{u} \simeq 2.18\right)$. (b) Maximal growth rate vs $\beta$. Thin (thick) lines correspond to $\delta=0(\delta=0.5)$. (c) Components of the eigenmode corresponding to the in-phase snake MI, $\beta=0.3, \delta=0$, $\Omega=1$. (d) Components of the eigenmode corresponding to the anti-phase snake MI, $\beta=0.3, \delta=0, \Omega=0.92$.

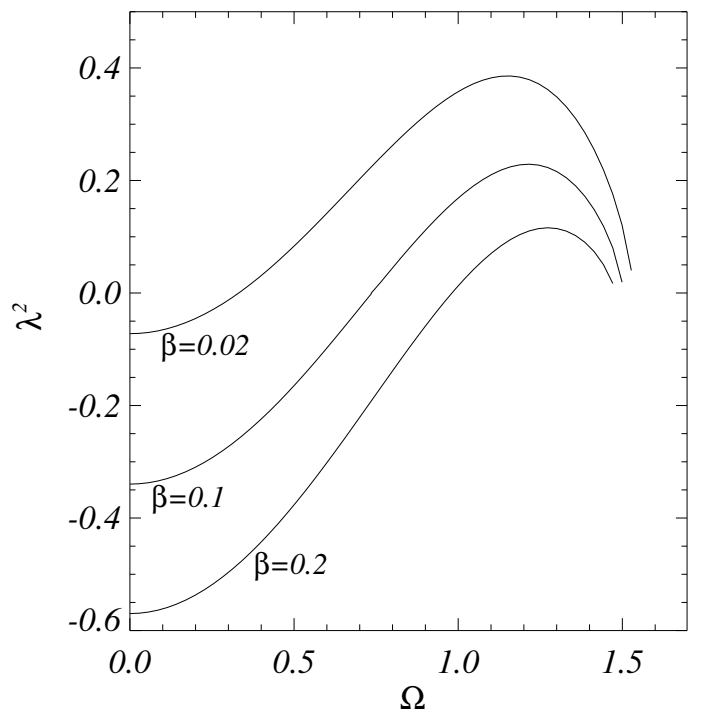

FIG. 5. Eigenvalues corresponding to the anti-phase snake MI vs $\Omega$ for several choices of $\beta: \kappa=1, \delta=0, \gamma=-0.5$.
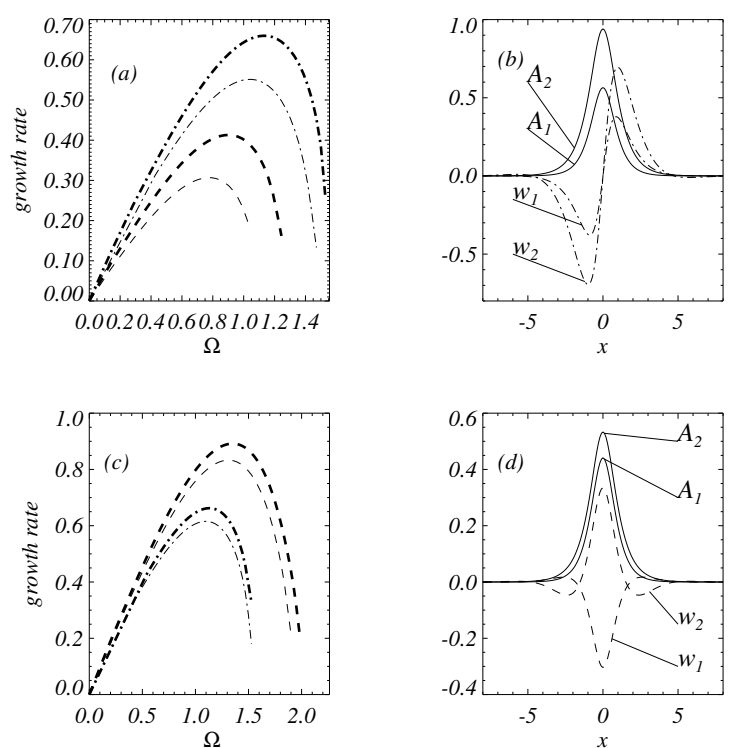

FIG. 6. Instability growth rates, spatial profiles of the solitary solutions and of the unstable eigenfunctions for $\beta>1$, $\gamma=-0.5$. Dash-dot (dash) lines correspond to in-phase snake (anti-phase neck) MIs. (a) Growth rates vs $\Omega, \beta=2$. Thin (thick) lines correspond to $\kappa=1, \delta=0, Q=1.75, Q_{u}=0$ $\left(\kappa=0.93, \delta=0.2, Q=1.75, Q_{u}=-0.91\right)$. (b) Components of the eigenmode corresponding to in-phase snake MI, $\beta=2, \delta=0.2, \Omega=0.8$. (c) Growth rates vs $\Omega, \beta=7$. Thin (thick) lines correspond to $\kappa=1, \delta=0, Q \simeq 0.68, Q_{u}=0$ $\left(\kappa=0.97, \delta=0.2, Q \simeq 0.68, Q_{u} \simeq-0.16\right)$. (d) Components of the eigenmode corresponding to anti-phase neck MI, $\beta=7$, $\delta=0.2, \Omega=1$.

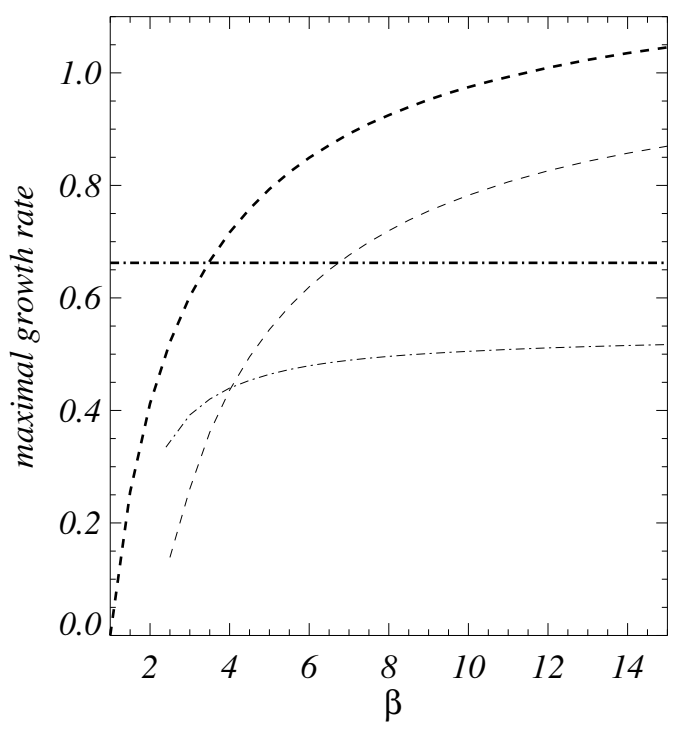

FIG. 7. Maximal growth rates of the in-phase snake (dash-dot line) and the anti-phase neck (dash line) MIs vs $\beta$ for $\beta>1, \gamma=-0.5$. Thin (thick) lines correspond to $\delta=0$ $(\delta=0.5)$. Cross-over occurs at $\beta_{s n} \simeq 3.47$ for $\delta=0$ and $\beta_{s n} \simeq 4.04$ for $\delta=0.5$. 

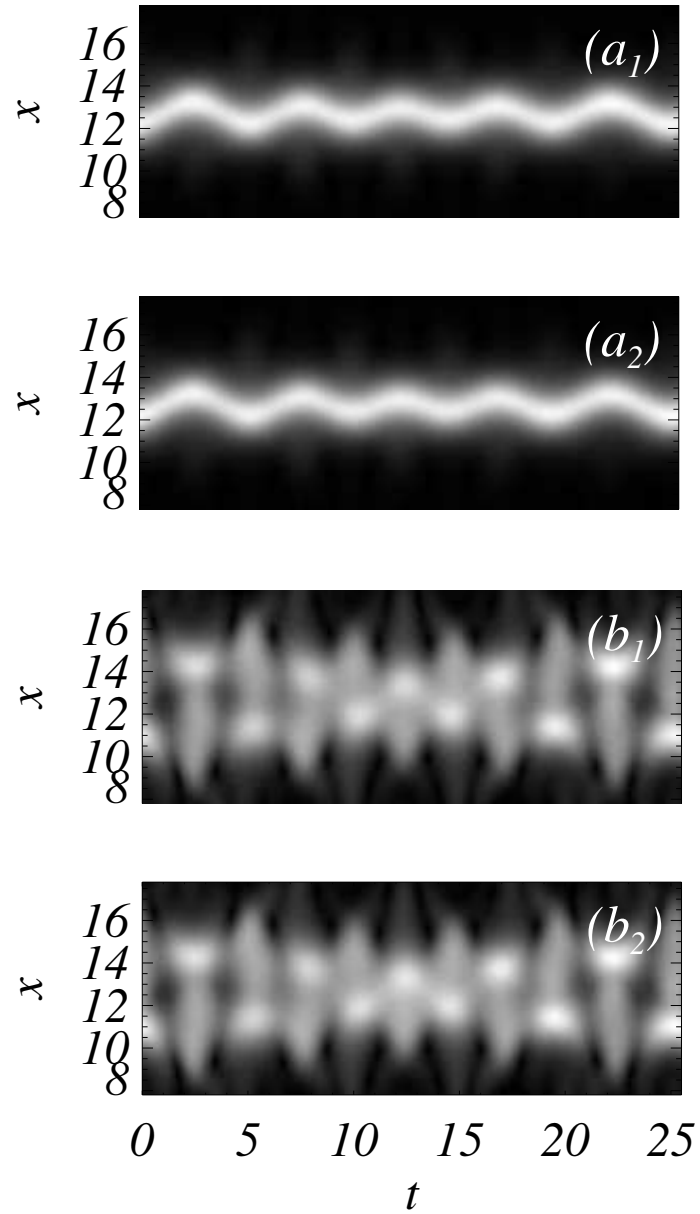

FIG. 8. Development of the in-phase snake MI for $\beta=2$, $\kappa=1, \delta=0, \gamma=-0.5$. $\left(\mathrm{a}_{1,2}\right)\left|E_{1,2}\right|$ for $z=12 ;\left(\mathrm{b}_{1,2}\right)\left|E_{1,2}\right|$ for $z=14.7$.
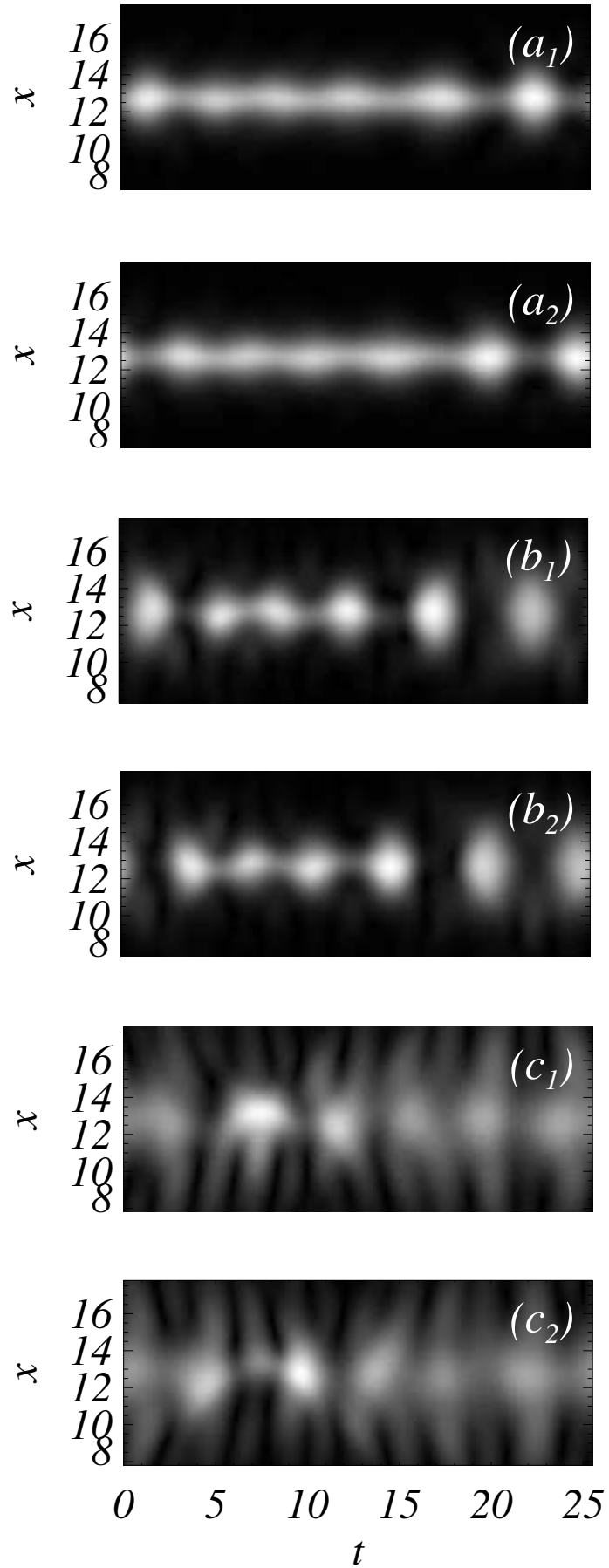

FIG. 9. Development of the anti-phase neck MI for $\beta=7$, $\kappa=1, \delta=0, \gamma=-0.5$. $\left(\mathrm{a}_{1,2}\right)\left|E_{1,2}\right|$ for $z=8.4 ;\left(\mathrm{b}_{1,2}\right)\left|E_{1,2}\right|$ for $z=10.2 ;\left(c_{1,2}\right)\left|E_{1,2}\right|$ for $z=12.6$. 

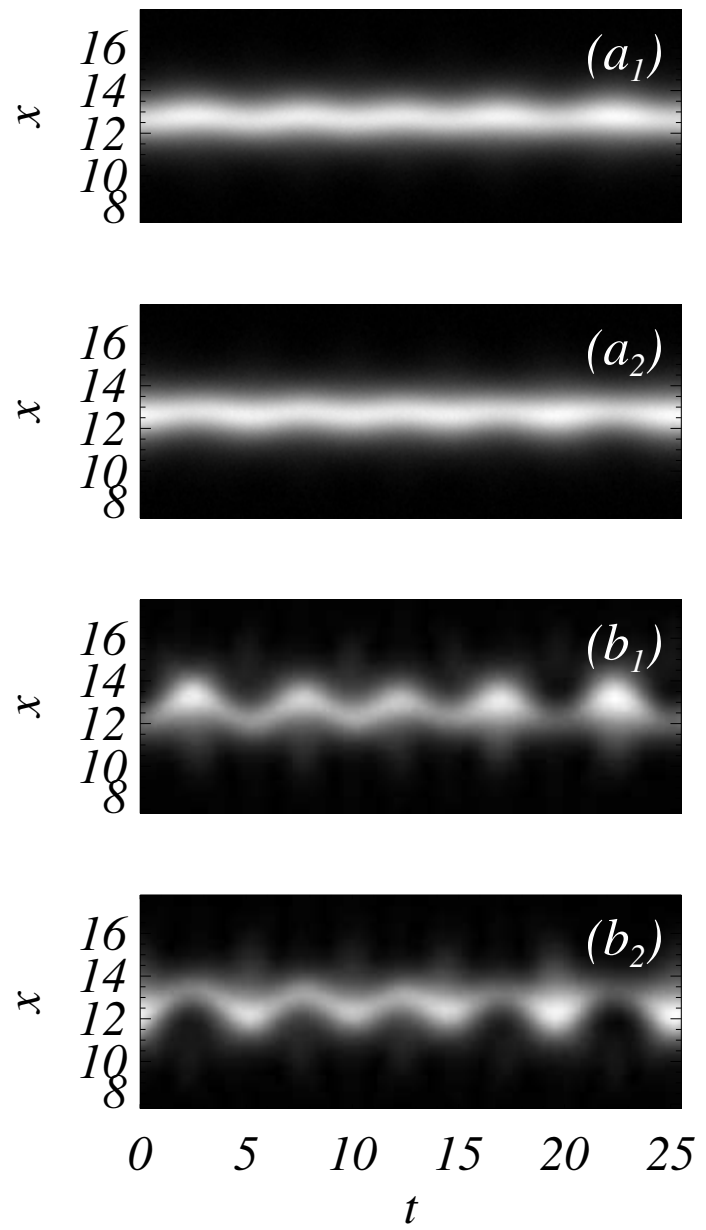

FIG. 10. Competition between the in-phase snake and anti-phase neck MIs: $\beta=3.47, \kappa=1, \delta=0, \gamma=-0.5$. $\left(\mathrm{a}_{1,2}\right)\left|E_{1,2}\right|$ for $z=9 ;\left(\mathrm{b}_{1,2}\right)\left|E_{1,2}\right|$ for $z=12$
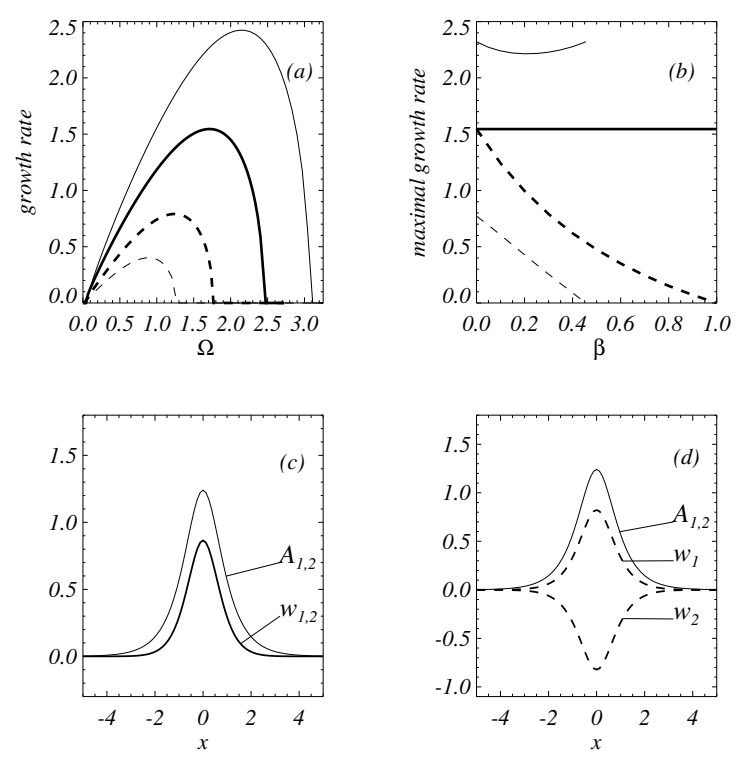

FIG. 11. Instability growth rates, spatial profiles of the solitary solutions and of the unstable eigenfunctions for $\beta<1$, $\gamma=0.5$. Full (dash) lines correspond to in-phase (anti-phase) neck MIs. (a) Growth rates vs $\Omega, \beta=0.3$. Thin (thick) lines correspond to $\kappa=1, \delta=0, Q \simeq 4.04, Q_{u}=0(\kappa=1.155$, $\delta=0.5, Q \simeq 4.04, Q_{u} \simeq 2.18$ ). (b) Maximal growth rate vs $\beta$. Thin (thick) lines correspond to $\delta=0(\delta=0.5)$. (c) Components of the eigenmode corresponding to in-phase snake MI, $\beta=0.3, \delta=0, \Omega=1.5$. (d) Componets of the eigenmode corresponding to anti-phase snake MI, $\beta=0.3, \delta=0$, $\Omega=0.9$.
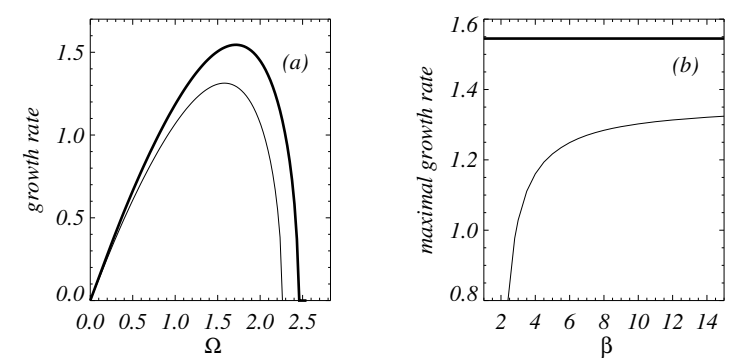

FIG. 12. In-phase MI growth rates for $\beta>1, \gamma=0.5$. (a) Growth rates vs $\Omega, \beta=2$. Thin (thick) lines correspond to $\kappa=1, \delta=0, Q \simeq 4.04, Q_{u}=0(\kappa=1.155, \delta=0.5$, $Q \simeq 4.04, Q_{u} \simeq 2.18$ ). (b) Maximal growth rate vs $\beta$. Thin (thick) lines correspond to $\delta=0(\delta=0.5)$.
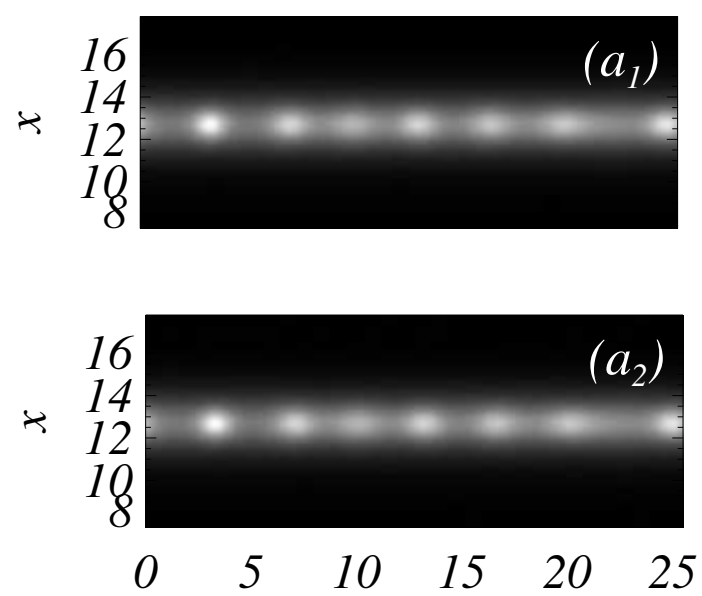

FIG. 13. Development of the in-phase neck MI for $\beta=2$, $\kappa=1, \delta=0, \gamma=0.5$. $\left(\mathrm{a}_{1,2}\right)\left|E_{1,2}\right|$ for $z=4$.6. With further increasing of $z$ most intense filaments develop collapse. 